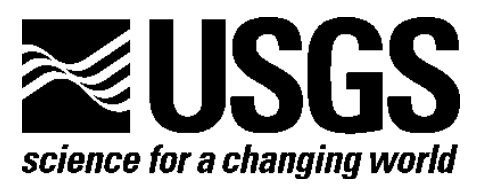

Summary of Chemical Data from Onsite and Laboratory Analyses of Groundwater Samples from the Surficial Aquifer, Las Vegas, Nevada, April and August 1993 and September 1994

Open-File Report 2012-1029 


\section{Summary of Chemical Data from Onsite and Laboratory Analyses of Groundwater Samples from the Surficial Aquifer, Las Vegas, Nevada, April and August 1993 and September 1994}

By Michael M. Reddy and Charmaine D. Gunther

Open-File Report 2012-1029 


\section{U.S. Department of the Interior \\ KEN SALAZAR, Secretary}

\section{U.S. Geological Survey \\ Marcia K. McNutt, Director}

U.S. Geological Survey, Reston, Virginia: 2012

For more information on the USGS-the Federal source for science about the Earth,

its natural and living resources, natural hazards, and the environment-visit

http://www.usgs.gov or call 1-888-ASK-USGS

For an overview of USGS information products, including maps, imagery, and publications, visit $h$ ttp://www.usgs.gov/pubprod

To order this and other USGS information products, visit $h$ ttp://store.usgs.gov

Suggested citation:

Reddy, M.M., and Gunther, C.D., 2012, Summary of chemical data from onsite and laboratory analyses of groundwater samples from the surficial aquifer, Las Vegas, Nevada, April and August 1993 and September 1994: U.S. Geological Survey Open-File Report 2012-1029, 18 p.

Any use of trade, product, or firm names is for descriptive purposes only and does not imply endorsement by the U.S. Government.

Although this report is in the public domain, permission must be secured from the individual copyright owners to reproduce any copyrighted material contained within this report. 


\section{Contents}

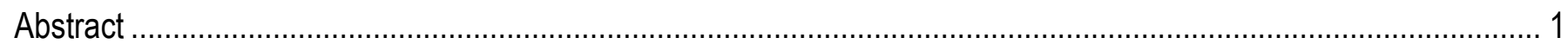

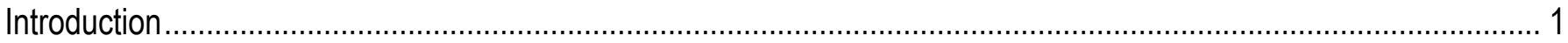

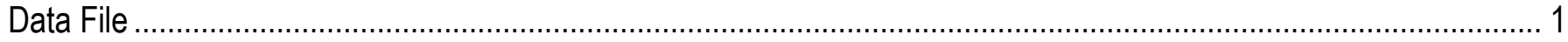

Summary

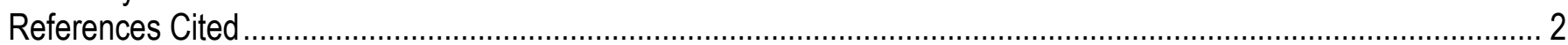

\section{Tables}

1. Variable names and codes for documenting sample collection........................................................................ 4

2. Definitions and units for onsite and laboratory measured variables ............................................................. 5

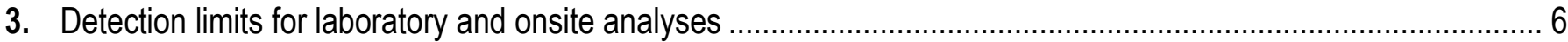

4. Summary statistics for standard reference water samples (SRWS) and distilled-water field blank (FB) and laboratory blank samples ........................................................................................................... 7

5. Chemical data for groundwater samples from the surficial aquifer, Las Vegas, Nev. $A$, Site locations and well information; $B$, Field parameters; $C$, Major ion chemistry; $D$, Trace constituents (Set A);

$E$, Trace constituents (Set B) 


\title{
Summary of Chemical Data from Onsite and Laboratory Analyses of Groundwater Samples from the Surficial Aquifer, Las Vegas, Nevada, April and August 1993 and September 1994
}

\author{
By Michael M. Reddy and Charmaine D. Gunther
}

\begin{abstract}
This report presents a summary of data collected during April and August 1993 and September 1994. These results are to be used as a wet-site analog to southern Nevada soils located at the Amargosa Desert Research Site near Beatty, Nevada. The samples were collected and analyzed in conjunction with the Nevada Basin and Range study unit of the U.S. Geological Survey, National Water-Quality Assessment Program (NAWQA).

Samples were collected from groundwater wells in and about the city of Las Vegas, Nevada, and were analyzed for selected major, minor and trace constituents. Analyses of blank and reference samples are summarized as mean and standard deviation values for all positive results.
\end{abstract}

\section{Introduction}

In 1993, 33 wells were chosen in and about the city of Las Vegas, Nevada, to determine the effects of land use on underlying water quality. Groundwater samples collected from these wells were analyzed for major, minor, and trace constituents. Details of the onsite and laboratory procedures were adapted from previous studies and are described by Reddy and others (1989). Sample preparation, analytical procedures, and laboratory quality-control protocols used by the U.S. Geological Survey laboratory are described by Fishman and Freidman (1985) Garbarino and Taylor (1979; 1980), and Schroder and others (1980). Onsite procedures and equipment were adapted from a trace metal protocol developed by the U.S. Geological Survey National Research Program (Taylor and others, 1995).

Sample collection and analyses were done in conjunction with the Nevada Basin and Range study unit of the U.S. Geological Survey, NAWQA (Emmett and others, 1993, and Clary and others, 1994).

\section{Data File}

Data are available from the analysis of 45 samples from 33 wells collected during 1993 and 1994. The data file consists of 37 variables. Variable names and codes established for sample collection are listed in table 1. Definitions and units for onsite and laboratory measured variables are listed in table 2. To evaluate onsite-sampling variability, duplicate samples were collected from some wells. Field replicate samples were analyzed to identify variability caused by sample processing and handling. Paired $t$ tests were used to assess differences due to filter pore size for each chemical species that had 
the adequate data. No statistically significant difference (at the $95 \%$ confidence level) was found between either duplicate field samples or between $0.4-\mu \mathrm{m}$ and $0.1-\mu \mathrm{m}$ filtered field samples for any chemical species. Duplicate $(0.4-\mu \mathrm{m}$ filtered) and $0.1-\mu \mathrm{m}$ filtered samples were averaged in the final data file. Additional distilled-water blank samples, referred to as field blanks (FB) and laboratory blanks (LB), and standard reference water samples (SRWS) ${ }^{1}$ were analyzed using the same procedures as the onsite samples. A check on the quality of the data was provided by a verification of summary statistics for the data, sorted by sample location. Table 3 reports the detection limits for all chemical constituents included in this report. Quality-assurance/quality-control statistics are shown on table 4. During 1993 and 1994, 45 samples were processed; analytical results are presented in table 5.

For further information, call Michael M. Reddy at (303) 235-5941 or E-mail mreddy@usgs.gov.

\section{Summary}

Forty-five samples from 33 groundwater wells were analyzed using a protocol designed to minimize errors from handling procedures, laboratory analyses, and data-entry operations. Analyses of blank samples and Standard Reference Water Sample results are included.

\section{References Cited}

Aiken, G.R., 1992, Chloride interference in the analysis of dissolved organic carbon by the wet oxidation method: Environmental Sciences and Technology, v. 26, p. 2435-2439

Clary, S.L., McClary, D.R., Whitney,R. And Reeves, D.D., 1995, Water resources data, Nevada, water year 1994: U.S. Geological Survey Water-Data Report NV-94-1, 768 p.

Crock, J.G., and Lichte, F.E., 1982, An improved method for the determination of trace-level arsenic and antimony in geological materials by automated hydride generation-atomic absorption spectroscopy: Analytica Chimica Acta, v. 144, p. 223-233.

Emett, D.C., Hutchinson, D.D., Jonson, N.A., O'Hair, K.L. 1993, Water resources data, Nevada, water year 1993: U.S. Geological Survey Water-Data Report NV-93-1, 596 p.

Fishman, M.J., and Friedman, L.C., 1985, Methods for determination of inorganic substances in water and fluvial sediments: U.S. Geological Survey Open-File Report 85-495, 709 p.

Garbarino, J.R., and Taylor, H.E., 1979, An inductive-coupled plasma atomic-emission spectrometric method for routine water quality testing: Applied Spectroscopy, v. 33, no. 3, p. 220-226.

Garbarino, J.R., and Taylor, H.E., 1980, A babington-type nebulizer for use in the analysis of natural water samples by inductively coupled plasma spectrometry: Applied Spectroscopy, v. 34, no. 5, p. 584-590.

Reddy, M.M., Schuster, P.F., and Harte, J.J., 1989, Summary of data from onsite and laboratory analyses of precipitation runoff from carbonate-stone surfaces, National Acid Precipitation Assessment Program, June 1984 to November 1987: U.S. Geological Survey Open-File Report 89-246, 19 p.

Schroder, L.J., Fishman, M.J., Friedman, L.C., and Darlington, G.W., 1980, The use of standard reference water samples by the U.S. Geological Survey: U.S. Geological Survey Open-File Report, 80-738, 14 p.

\footnotetext{
${ }^{1}$ Standard Reference Water Samples are prepared and used by the U.S. Geological Survey's quality assurance program to ensure that the laboratory is producing analytical data for inorganic constituents that are of acceptable reliability (Schroder and others, 1980)
} 
Skougstad, M.W., Fishman, M.J., Friedman, L.C., Erdmann, D.E., and Duncan, S.S., 1979, Methods for the determination of inorganic substances in water and fluvial sediments: U.S. Geological Survey Techniques of Water-Resources Investigations, book 5, chap. A1, 626 p.

Taylor, H.E., Shiller, A.M., Garbarino, J.R. and Brinton, T.I., 1995, Intercomparison experiments on dissolved trace-metal data from the Mississippi River and some of its tributaries, 1989-90: U.S. Geological Survey Open-File Report 93-628, 28 p.

Welsch, E.P., Crock, J.G., and Sanzolone, R., 1990, Trace-level determination arsenic and selenium using continuous-flow hydride generation atomic absorption spectrophotometry (HG-AAS): U.S. Geological Survey Open-File Report 90-668, p. 38-45.

Wood, W.W., 1976, Guidelines for collection and field analysis of ground-water samples for selected unstable constituents: Techniques of Water-Resources Investigation of the U.S. Geological Survey 01-D2, $24 \mathrm{p}$. 
Table 1. Variable names and codes for documenting sample collection.

\begin{tabular}{lll}
\hline \multicolumn{1}{c}{ Variable name } & \multicolumn{1}{c}{ Code } & \multicolumn{1}{c}{ Definition } \\
\hline Site ID & 15-digit integer & Site identification number \\
Site name & alphanumeric string & Short description of site \\
Latitude (DDMMSS) & 6-digit integer & Latitude (degrees minutes seconds) \\
Longitude (DDDMMSS) & 7-digit integer & Longitude (degrees minutes seconds) \\
Altitude (feet) & integer & Altitude of top of casing, in feet \\
Well depth (feet) & floating point & Well depth from top of casing, in feet \\
Depth to water (feet) & floating point & Depth to water from top of casing, in feet \\
Screen interval (feet) & alphanumeric string & Screened interval of well from top of casing, \\
Collection date (mm/dd/yy) & alphanumeric string & in feet \\
\hline
\end{tabular}

Download Comma Separated Values (table1.csv $<1 \mathrm{k}$ ) 
Table 2. Definitions and units for onsite and laboratory measured variables.

\begin{tabular}{|c|c|c|}
\hline Variable name & Definition & Units \\
\hline Alkalinity $(\mathrm{meq} / \mathrm{L})$ & alkalinity measured in laboratory & milliequivalents per liter \\
\hline As $(\mu \mathrm{g} / \mathrm{L})$ & dissolved arsenic as As & micrograms per liter \\
\hline $\mathrm{B}(\mathrm{mg} / \mathrm{L})$ & dissolved boron as B & milligrams per liter \\
\hline $\mathrm{Ba}(\mathrm{mg} / \mathrm{L})$ & dissolved barium as $\mathrm{Ba}$ & milligrams per liter \\
\hline $\mathrm{Be}(\mathrm{mg} / \mathrm{L})$ & dissolved beryllium as $\mathrm{Be}$ & milligrams per liter \\
\hline $\mathrm{Ca}(\mathrm{meq} / \mathrm{L})$ & dissolved calcium as $\mathrm{Ca}$ & milliequivalents per liter \\
\hline $\mathrm{Cd}(\mathrm{mg} / \mathrm{L})$ & dissolved cadmium as $\mathrm{Cd}$ & milligrams per liter \\
\hline $\mathrm{Cl}(\mathrm{meq} / \mathrm{L})$ & dissolved chloride as $\mathrm{Cl}$ & milliequivalents per liter \\
\hline $\mathrm{Co}(\mathrm{mg} / \mathrm{L})$ & dissolved cobalt as Co & milligrams per liter \\
\hline $\mathrm{Cu}(\mathrm{mg} / \mathrm{L})$ & dissolved copper as $\mathrm{Cu}$ & milligrams per liter \\
\hline $\mathrm{DOC}(\mathrm{mg} / \mathrm{L})$ & dissolved organic carbon as $\mathrm{C}$ & milligrams per liter \\
\hline FB & field blank & none \\
\hline $\mathrm{Fe}(\mathrm{mg} / \mathrm{L})$ & dissolved iron as $\mathrm{Fe}$ & milligrams per liter \\
\hline Field dissolved oxygen (mg/L) & dissolved oxygen measured onsite & milligrams per liter \\
\hline Field pH (std units) & $\mathrm{pH}$ measured onsite & standard $\mathrm{pH}$ units \\
\hline Field specific conductivity $(\mu \mathrm{S} / \mathrm{cm})$ & specific conductivity measured onsite & microsiemens per centimeter at $25^{\circ} \mathrm{C}$ \\
\hline Ion balance $(\%)$ & $100 \% \times($ cations - anions $) /($ cations + anions $)$ & $(+/-)$ percent \\
\hline $\mathrm{K}(\mathrm{meq} / \mathrm{L})$ & dissolved potassium as $\mathrm{K}$ & milliequivalents per liter \\
\hline LB & laboratory blank & none \\
\hline $\mathrm{Li}(\mathrm{mg} / \mathrm{L})$ & dissolved lithium as Li & milligrams per liter \\
\hline M106 & major ion SRWS & none \\
\hline $\mathrm{Mg}(\mathrm{meq} / \mathrm{L})$ & dissolved magnesium as $\mathrm{Mg}$ & milliequivalents per liter \\
\hline $\operatorname{Mn}(\mathrm{mg} / \mathrm{L})$ & dissolved manganese as $\mathrm{Mn}$ & milligrams per liter \\
\hline $\mathrm{Na}(\mathrm{meq} / \mathrm{L})$ & dissolved sodium as $\mathrm{Na}$ & milliequivalents per liter \\
\hline $\operatorname{Se}(\mu \mathrm{g} / \mathrm{L})$ & dissolved selenium as $\mathrm{Se}$ & micrograms per liter \\
\hline $\mathrm{SiO}_{2}(\mathrm{mg} / \mathrm{L})$ & dissolved silica as $\mathrm{SiO}_{2}$ & milligrams per liter \\
\hline $\mathrm{SO}_{4}(\mathrm{meq} / \mathrm{L})$ & dissolved sulfate as $\mathrm{SO}_{4}$ & milliequivalents per liter \\
\hline $\mathrm{Sr}(\mathrm{mg} / \mathrm{L})$ & dissolved strontium as $\mathrm{Sr}$ & milligrams per liter \\
\hline SRWS & Standard Reference Water Samples & none \\
\hline T107 & trace metal SRWS & none \\
\hline $\mathrm{V}(\mathrm{mg} / \mathrm{L})$ & dissolved vanadium as $\mathrm{V}$ & milligrams per liter \\
\hline Water temp $\left({ }^{\circ} \mathrm{C}\right)$ & water temperature measured onsite & degrees Celsius \\
\hline $\mathrm{Zn}(\mathrm{mg} / \mathrm{L})$ & dissolved zinc as $\mathrm{Zn}$ & milligrams per liter \\
\hline
\end{tabular}

Download Comma Separated Values (table2.csv 2k) 
Table 3. Detection limits for laboratory and onsite analyses. (meq/L, milliequivalents per liter; $\mu \mathrm{g} / \mathrm{L}$, micrograms per liter; $\mathrm{mg} / \mathrm{L}$, milligrams per liter)

\begin{tabular}{ccccl}
\hline Constituent & Units & $\begin{array}{c}\text { Typical } \\
\text { dilution }\end{array}$ & $\begin{array}{c}\text { Detection } \\
\text { limit }\end{array}$ & \multicolumn{1}{c}{ Method of analysis } \\
\hline Alkalinity & $(\mathrm{meq} / \mathrm{L})$ & none & none & Fishman and Friedman, 1985 \\
$\mathrm{As}$ & $(\mu \mathrm{g} / \mathrm{L})$ & none & 2 & Crock and Lichte, 1982 and Welsch and others, 1990 \\
$\mathrm{B}$ & $(\mathrm{mg} / \mathrm{L})$ & none & .002 & Garbarino and Taylor, 1979, 1980 \\
$\mathrm{Ba}$ & $(\mathrm{mg} / \mathrm{L})$ & none & .001 & Garbarino and Taylor, 1979, 1980 \\
$\mathrm{Be}$ & $(\mathrm{mg} / \mathrm{L})$ & none & .001 & Garbarino and Taylor, 1979, 1980 \\
$\mathrm{Ca}$ & $(\mathrm{meq} / \mathrm{L})$ & $1: 10$ & .0005 & Garbarino and Taylor, 1979, 1980 \\
$\mathrm{Cd}$ & $(\mathrm{mg} / \mathrm{L})$ & none & .01 & Garbarino and Taylor, 1979, 1980 \\
$\mathrm{Cl}$ & $(\mathrm{meq} / \mathrm{L})$ & $1: 100$ & .2 & Fishman and Friedman, 1985 \\
$\mathrm{Co}$ & $(\mathrm{mg} / \mathrm{L})$ & None & .01 & Garbarino and Taylor, 1979, 1980 \\
$\mathrm{Cu}$ & $(\mathrm{mg} / \mathrm{L})$ & None & .005 & Garbarino and Taylor, 1979, 1980 \\
$\mathrm{DO}$ & $(\mathrm{mg} / \mathrm{L})$ & None & .1 & Wood, 1976 \\
$\mathrm{DOC}$ & $(\mathrm{mg} / \mathrm{L})$ & None & .1 & Aiken, 1992 \\
$\mathrm{Fe}$ & $(\mathrm{mg} / \mathrm{L})$ & None & .005 & Garbarino and Taylor, 1979, 1980 \\
$\mathrm{K}$ & $(\mathrm{meq} / \mathrm{L})$ & $1: 10$ & .01 & Skougstad and others, 1979 \\
$\mathrm{Li}$ & $(\mathrm{mg} / \mathrm{L})$ & none & .005 & Garbarino and Taylor, 1979, 1980 \\
$\mathrm{Mg}$ & $(\mathrm{meq} / \mathrm{L})$ & $1: 10$ & .0008 & Garbarino and Taylor, 1979, 1980 \\
$\mathrm{Mn}$ & $(\mathrm{mg} / \mathrm{L})$ & None & .002 & Garbarino and Taylor, 1979, 1980 \\
$\mathrm{Na}$ & $(\mathrm{meq} / \mathrm{L})$ & $1: 10$ & .004 & Garbarino and Taylor, 1979, 1980 \\
$\mathrm{Se}$ & $(\mu \mathrm{g} / \mathrm{L})$ & none & 1 & Crock and Lichte, 1982 and Welsch and others, 1990 \\
$\mathrm{SiO}{ }_{2}$ & $(\mathrm{mg} / \mathrm{L})$ & none & .04 & Garbarino and Taylor, 1979, 1980 \\
$\mathrm{SO} \mathrm{H}_{4}$ & $(\mathrm{meq} / \mathrm{L})$ & $1: 100$ & 2 & Fishman and Friedman, 1985 \\
$\mathrm{Sr}$ & $(\mathrm{mg} / \mathrm{L})$ & none & .001 & Garbarino and Taylor, 1979, 1980 \\
$\mathrm{V}$ & $(\mathrm{mg} / \mathrm{L})$ & none & .005 & Garbarino and Taylor, 1979, 1980 \\
$\mathrm{Zn}$ & $(\mathrm{mg} / \mathrm{L})$ & none & .01 & Garbarino and Taylor, 1979, 1980 \\
\hline & & & &
\end{tabular}

Download Comma Separated Values(table3.csv 1k) 
Table 4. Summary statistics for standard reference water samples (SRWS) and distilled-water field blank (FB) and laboratory blank samples. (meq/L, milliequivalents per liter; $\mu \mathrm{g} / \mathrm{L}$, micrograms per liter; mg/L, milligrams per liter; N/A, not applicable; FB, field blank; LB, laboratory blank).-Continued

\begin{tabular}{|c|c|c|c|c|c|c|c|c|c|c|c|}
\hline \multirow[b]{2}{*}{ Constituent } & \multirow[b]{2}{*}{ Sample } & \multirow[b]{2}{*}{ Units } & \multirow[b]{2}{*}{$\begin{array}{l}\text { Mean of } \\
\text { reported } \\
\text { values }\end{array}$} & \multirow[b]{2}{*}{$\begin{array}{l}\text { Standard } \\
\text { deviation }\end{array}$} & \multicolumn{2}{|c|}{ Range } & \multicolumn{2}{|c|}{$\begin{array}{l}\text { Number of } \\
\text { samples }\end{array}$} & \multicolumn{3}{|c|}{$\begin{array}{c}\text { Expected value } \\
\text { (Schroder and others 1980) }\end{array}$} \\
\hline & & & & & Minimum & Maximum & $\begin{array}{l}\text { Less than } \\
\text { detection }\end{array}$ & $\mathrm{N}$ & $\begin{array}{c}95 \% \\
\text { confidence } \\
\text { MPV }\end{array}$ & $\begin{array}{l}\text { Standard } \\
\text { deviation }\end{array}$ & $\mathrm{N}$ \\
\hline \multirow[t]{3}{*}{ Alkalinity } & FB & $(\mathrm{meq} / \mathrm{L})$ & -0.03 & 0.05 & -0.106 & -0.001 & 0 & 4 & & & \\
\hline & LB & $(\mathrm{meq} / \mathrm{L})$ & -.002 & .0013 & -.004 & -.001 & 0 & 6 & & & \\
\hline & M106 & $(\mathrm{meq} / \mathrm{L})$ & .112 & .001 & .109 & .114 & 0 & 12 & $.16 \pm 0$ & .022 & 55 \\
\hline \multirow[t]{2}{*}{ As } & FB & $(\mu \mathrm{g} / \mathrm{L})$ & $<2$ & N/A & $<2$ & $<2$ & 4 & 4 & & & \\
\hline & T107 & $(\mu \mathrm{g} / \mathrm{L})$ & 11 & .8 & 10 & 12 & 0 & 4 & $1.8 \pm 1$ & 2.1 & 57 \\
\hline \multirow[t]{4}{*}{ B } & FB & $(\mathrm{mg} / \mathrm{L})$ & $<.002$ & N/A & $<.002$ & $<.002$ & 2 & 2 & & & \\
\hline & LB & $(\mathrm{mg} / \mathrm{L})$ & .003 & N/A & .003 & .003 & 13 & 14 & & & \\
\hline & M106 & $(\mathrm{mg} / \mathrm{L})$ & .042 & .047 & .011 & .178 & 1 & 17 & $.044 \pm 0$ & .037 & 18 \\
\hline & $\mathrm{T} 107$ & $(\mathrm{mg} / \mathrm{L})$ & .125 & .014 & .092 & .149 & 0 & 18 & $.13 \pm 0$ & .021 & 37 \\
\hline \multirow[t]{3}{*}{$\mathrm{Ba}$} & FB & $(\mathrm{mg} / \mathrm{L})$ & $<.001$ & N/A & $<.001$ & $<.001$ & 2 & 2 & & & \\
\hline & LB & $(\mathrm{mg} / \mathrm{L})$ & .002 & 0 & .002 & .002 & 11 & 14 & & & \\
\hline & T107 & $(\mathrm{mg} / \mathrm{L})$ & .189 & .014 & .157 & .209 & 0 & 18 & $.192 \pm 0$ & .011 & 53 \\
\hline \multirow[t]{3}{*}{$\mathrm{Be}$} & $\mathrm{FB}$ & $(\mathrm{mg} / \mathrm{L})$ & $<.001$ & N/A & $<.001$ & $<.001$ & 2 & 2 & & & \\
\hline & LB & $(\mathrm{mg} / \mathrm{L})$ & .002 & .001 & .001 & .003 & 9 & 14 & & & \\
\hline & T107 & $(\mathrm{mg} / \mathrm{L})$ & .01 & .004 & .003 & .016 & 1 & 18 & $.011 \pm 0$ & .0011 & 34 \\
\hline \multirow[t]{4}{*}{$\mathrm{Ca}$} & $\mathrm{FB}$ & $(\mathrm{meq} / \mathrm{L})$ & .0016 & N/A & .0016 & .0016 & 1 & 2 & & & \\
\hline & LB & $(\mathrm{meq} / \mathrm{L})$ & .0006 & .0003 & .0001 & .001 & 4 & 14 & & & \\
\hline & M106 & $(\mathrm{meq} / \mathrm{L})$ & .45 & .03 & .41 & .51 & 0 & 17 & $.45 \pm 0$ & .02 & 62 \\
\hline & $\mathrm{T} 107$ & $(\mathrm{meq} / \mathrm{L})$ & .57 & .04 & .47 & .63 & 0 & 18 & $.584 \pm 0$ & .03 & 60 \\
\hline \multirow[t]{3}{*}{$\mathrm{Cd}$} & FB & $(\mathrm{mg} / \mathrm{L})$ & $<.01$ & N/A & $<.01$ & $<.01$ & 2 & 2 & & & \\
\hline & LB & $(\mathrm{mg} / \mathrm{L})$ & .014 & .005 & .01 & .021 & 10 & 14 & & & \\
\hline & T107 & $(\mathrm{mg} / \mathrm{L})$ & .014 & .004 & .01 & .025 & 5 & 18 & $.0143 \pm 0$ & .0021 & 70 \\
\hline \multirow[t]{2}{*}{$\mathrm{Cl}$} & FB & $(\mathrm{meq} / \mathrm{L})$ & .05 & .09 & .001 & .188 & 0 & 4 & & & \\
\hline & LB & $(\mathrm{meq} / \mathrm{L})$ & .003 & .002 & .002 & .004 & 66 & 68 & & & \\
\hline
\end{tabular}


Table 4. Summary statistics for standard reference water samples (SRWS) and distilled-water field blank (FB) and laboratory blank samples. (meq/L, milliequivalents per liter; $\mu \mathrm{g} / \mathrm{L}$, micrograms per liter; mg/L, milligrams per liter; N/A, not applicable; FB, field blank; LB, laboratory blank).-Continued

\begin{tabular}{|c|c|c|c|c|c|c|c|c|c|c|c|}
\hline \multirow[b]{2}{*}{ Constituent } & \multirow[b]{2}{*}{ Sample } & \multirow[b]{2}{*}{ Units } & \multirow[b]{2}{*}{$\begin{array}{l}\text { Mean of } \\
\text { reported } \\
\text { values }\end{array}$} & \multirow[b]{2}{*}{$\begin{array}{l}\text { Standard } \\
\text { deviation }\end{array}$} & \multicolumn{2}{|c|}{ Range } & \multicolumn{2}{|c|}{$\begin{array}{l}\text { Number of } \\
\text { samples }\end{array}$} & \multicolumn{3}{|c|}{$\begin{array}{c}\text { Expected value } \\
\text { (Schroder and others 1980) }\end{array}$} \\
\hline & & & & & Minimum & Maximum & $\begin{array}{l}\text { Less than } \\
\text { detection }\end{array}$ & $\mathrm{N}$ & $\begin{array}{c}95 \% \\
\text { confidence } \\
\text { MPV }\end{array}$ & $\begin{array}{l}\text { Standard } \\
\text { deviation }\end{array}$ & $\mathrm{N}$ \\
\hline \multirow{3}{*}{$\mathrm{Co}$} & M106 & $(\mathrm{meq} / \mathrm{L})$ & .39 & .03 & .32 & .423 & 0 & 12 & $.37 \pm 0$ & .034 & 68 \\
\hline & FB & $(\mathrm{mg} / \mathrm{L})$ & $<.01$ & N/A & $<.01$ & $<.01$ & 2 & 2 & & & \\
\hline & LB & $(\mathrm{mg} / \mathrm{L})$ & $<.01$ & N/A & $<.01$ & $<.01$ & 14 & 14 & & & \\
\hline \multirow{3}{*}{$\mathrm{Cu}$} & $\mathrm{T} 107$ & $(\mathrm{mg} / \mathrm{L})$ & .014 & .003 & .011 & .019 & 6 & 18 & $.011 \pm 0$ & .0014 & 34 \\
\hline & $\mathrm{FB}$ & $(\mathrm{mg} / \mathrm{L})$ & $<.005$ & N/A & $<.005$ & $<.005$ & 2 & 2 & & & \\
\hline & LB & $(\mathrm{mg} / \mathrm{L})$ & .017 & 0 & .017 & .017 & 12 & 14 & & & \\
\hline \multirow{3}{*}{$\mathrm{Fe}$} & T107 & $(\mathrm{mg} / \mathrm{L})$ & .026 & .007 & .011 & .035 & 0 & 18 & $.03 \pm 0$ & .0023 & 73 \\
\hline & FB & $(\mathrm{mg} / \mathrm{L})$ & $<.005$ & N/A & $<.005$ & $<.005$ & 2 & 2 & & & \\
\hline & $\mathrm{LB}$ & $(\mathrm{mg} / \mathrm{L})$ & .02 & .008 & .012 & .029 & 6 & 14 & & & \\
\hline \multirow{3}{*}{ K } & $\mathrm{T} 107$ & $(\mathrm{mg} / \mathrm{L})$ & .04 & .028 & .012 & .102 & 1 & 18 & $.052 \pm 0$ & .007 & 68 \\
\hline & $\mathrm{FB}$ & $(\mathrm{meq} / \mathrm{L})$ & .02 & .02 & .002 & .031 & 2 & 6 & & & \\
\hline & M106 & $(\mathrm{meq} / \mathrm{L})$ & .017 & .003 & .014 & .023 & 0 & 5 & $.02 \pm 0$ & .003 & 58 \\
\hline \multirow{3}{*}{$\mathrm{Li}$} & $\mathrm{T} 107$ & $(\mathrm{meq} / \mathrm{L})$ & .023 & .004 & .019 & .028 & 0 & 4 & $.021 \pm 0$ & .0038 & 62 \\
\hline & $\mathrm{FB}$ & $(\mathrm{mg} / \mathrm{L})$ & $<.005$ & N/A & $<.005$ & $<.005$ & 2 & 2 & & & \\
\hline & LB & $(\mathrm{mg} / \mathrm{L})$ & $<.005$ & N/A & $<.005$ & $<.005$ & 14 & 14 & & & \\
\hline \multirow{3}{*}{$\mathrm{Mg}$} & $\mathrm{T} 107$ & $(\mathrm{mg} / \mathrm{L})$ & .18 & .03 & .1 & .244 & 0 & 18 & $.193 \pm 0$ & .014 & 21 \\
\hline & FB & $(\mathrm{meq} / \mathrm{L})$ & .0002 & N/A & .0002 & .0002 & 1 & 2 & & & \\
\hline & LB & $(\mathrm{meq} / \mathrm{L})$ & .0009 & .0008 & .0001 & .0032 & 1 & 14 & & & \\
\hline \multirow{5}{*}{$\mathrm{Mn}$} & M106 & $(\mathrm{meq} / \mathrm{L})$ & .282 & .013 & .253 & .301 & 0 & 17 & $.29 \pm 0$ & .02 & 62 \\
\hline & $\mathrm{T} 107$ & $(\mathrm{meq} / \mathrm{L})$ & .168 & .01 & .139 & .179 & 0 & 18 & $.173 \pm 0$ & .011 & 61 \\
\hline & $\mathrm{FB}$ & $(\mathrm{mg} / \mathrm{L})$ & $<.002$ & N/A & $<.002$ & $<.002$ & 2 & 2 & & & \\
\hline & LB & $(\mathrm{mg} / \mathrm{L})$ & $<.002$ & N/A & $<.002$ & $<.002$ & 14 & 14 & & & \\
\hline & T107 & $(\mathrm{mg} / \mathrm{L})$ & .047 & .003 & .039 & .052 & 0 & 18 & $.045 \pm 0$ & .006 & 68 \\
\hline $\mathrm{Na}$ & $\mathrm{FB}$ & $(\mathrm{meq} / \mathrm{L})$ & .005 & N/A & .005 & .005 & 1 & 2 & & & \\
\hline
\end{tabular}


Table 4. Summary statistics for standard reference water samples (SRWS) and distilled-water field blank (FB) and laboratory blank samples. (meq/L, milliequivalents per liter; $\mu \mathrm{g} / \mathrm{L}$, micrograms per liter; mg/L, milligrams per liter; N/A, not applicable; FB, field blank; LB, laboratory blank).-Continued

\begin{tabular}{|c|c|c|c|c|c|c|c|c|c|c|c|}
\hline \multirow[b]{2}{*}{ Constituent } & \multirow[b]{2}{*}{ Sample } & \multirow[b]{2}{*}{ Units } & \multirow[b]{2}{*}{$\begin{array}{l}\text { Mean of } \\
\text { reported } \\
\text { values }\end{array}$} & \multirow[b]{2}{*}{$\begin{array}{l}\text { Standard } \\
\text { deviation }\end{array}$} & \multicolumn{2}{|c|}{ Range } & \multicolumn{2}{|c|}{$\begin{array}{l}\text { Number of } \\
\text { samples }\end{array}$} & \multicolumn{3}{|c|}{$\begin{array}{c}\text { Expected value } \\
\text { (Schroder and others 1980) }\end{array}$} \\
\hline & & & & & Minimum & Maximum & $\begin{array}{l}\text { Less than } \\
\text { detection }\end{array}$ & $\mathbf{N}$ & $\begin{array}{c}95 \% \\
\text { confidence } \\
\text { MPV }\end{array}$ & $\begin{array}{l}\text { Standard } \\
\text { deviation }\end{array}$ & $\mathrm{N}$ \\
\hline \multirow{5}{*}{$\mathrm{Se}$} & LB & $(\mathrm{meq} / \mathrm{L})$ & .0011 & .0011 & .0005 & .004 & 4 & 14 & & & \\
\hline & M106 & $(\mathrm{meq} / \mathrm{L})$ & .38 & .07 & .22 & .509 & 1 & 17 & $.4 \pm 0$ & .02 & 65 \\
\hline & $\mathrm{T} 107$ & $(\mathrm{meq} / \mathrm{L})$ & .8 & .2 & .423 & 1.11 & 0 & 18 & $.9 \pm 0$ & .048 & 65 \\
\hline & $\mathrm{FB}$ & $(\mu \mathrm{g} / \mathrm{L})$ & $<1$ & N/A & $<1$ & $<1$ & 4 & 4 & & & \\
\hline & T107 & $(\mu \mathrm{g} / \mathrm{L})$ & 13 & .8 & 12 & 14 & 0 & 4 & $11 \pm 1$ & 1.9 & 54 \\
\hline \multirow[t]{4}{*}{$\mathrm{SiO}_{2}$} & FB & $(\mathrm{mg} / \mathrm{L})$ & $<.04$ & N/A & $<.04$ & $<.04$ & 2 & 2 & & & \\
\hline & LB & $(\mathrm{mg} / \mathrm{L})$ & $<.04$ & N/A & $<.04$ & $<.04$ & 14 & 14 & & & \\
\hline & M106 & $(\mathrm{mg} / \mathrm{L})$ & 4 & 1 & 2.7 & 7.1 & 0 & 17 & $3.4 \pm 0$ & .2 & 40 \\
\hline & $\mathrm{T} 107$ & $(\mathrm{mg} / \mathrm{L})$ & 7.6 & 1 & 5.25 & 9.15 & 0 & 18 & $7.7 \pm 0$ & .5 & 43 \\
\hline \multirow[t]{3}{*}{$\mathrm{SO}_{4}$} & FB & $(\mathrm{meq} / \mathrm{L})$ & .289 & .001 & .288 & .289 & 2 & 4 & & & \\
\hline & LB & $(\mathrm{meq} / \mathrm{L})$ & .03 & $\mathrm{~N} / \mathrm{A}$ & .03 & .03 & 67 & 68 & & & \\
\hline & M106 & $(\mathrm{meq} / \mathrm{L})$ & .583 & .003 & .579 & .586 & 6 & 12 & $.575 \pm 0$ & .035 & 61 \\
\hline \multirow[t]{4}{*}{$\mathrm{Sr}$} & FB & $(\mathrm{mg} / \mathrm{L})$ & $<.001$ & N/A & $<.001$ & $<.001$ & 2 & 2 & & & \\
\hline & LB & $(\mathrm{mg} / \mathrm{L})$ & .0011 & .0001 & .001 & .001 & 11 & 14 & & & \\
\hline & M106 & $(\mathrm{mg} / \mathrm{L})$ & .16 & .04 & .019 & .194 & 0 & 17 & $.177 \pm 0$ & .009 & 22 \\
\hline & $\mathrm{T} 107$ & $(\mathrm{mg} / \mathrm{L})$ & .059 & .005 & .046 & .068 & 0 & 18 & $.061 \pm 0$ & .004 & 29 \\
\hline \multirow[t]{4}{*}{$\mathrm{V}$} & $\mathrm{FB}$ & $(\mathrm{mg} / \mathrm{L})$ & $<.005$ & N/A & $<.005$ & $<.005$ & 2 & 2 & & & \\
\hline & LB & $(\mathrm{mg} / \mathrm{L})$ & $<.005$ & N/A & $<.005$ & $<.005$ & 14 & 14 & & & \\
\hline & M106 & $(\mathrm{mg} / \mathrm{L})$ & .0063 & .0011 & .005 & .008 & 13 & 17 & $.0044 \pm 0$ & .0018 & 4 \\
\hline & $\mathrm{T} 107$ & $(\mathrm{mg} / \mathrm{L})$ & .014 & .003 & .007 & .018 & 0 & 18 & $.014 \pm 0$ & .0028 & 29 \\
\hline \multirow[t]{3}{*}{$\mathrm{Zn}$} & $\mathrm{FB}$ & $(\mathrm{mg} / \mathrm{L})$ & .01 & N/A & .01 & .01 & 1 & 2 & & & \\
\hline & LB & $(\mathrm{mg} / \mathrm{L})$ & .03 & .01 & .02 & .04 & 8 & 14 & & & \\
\hline & T107 & $(\mathrm{mg} / \mathrm{L})$ & .08 & .02 & .04 & .13 & 0 & 18 & $.0758 \pm 0$ & .0099 & 68 \\
\hline
\end{tabular}

Download Comma Separated Values (table4.csv 4k) 
Table 5. Chemical data for groundwater samples from the surficial aquifer, Las Vegas, Nev. A, Site locations and well information; B, Field parameters; C, Major ion chemistry; D, Trace constituents (Set A); E, Trace constituents (Set B). (D, degrees; M, minutes; $S$, seconds; $\mathrm{mg} / \mathrm{L}$, milligrams per liter; std, standard deviation; $\mu \mathrm{S} / \mathrm{cm}$, microseimens per centimeter; ${ }^{\circ} \mathrm{C}$, degrees Celsius; meq/L, milliequivalents per liter; $\mu \mathrm{g} / \mathrm{L}$, micrograms per liter; $\mathrm{NS}$, not sampled).-Continued

Table 5A, Site locations and well information

\begin{tabular}{|c|c|c|c|c|c|c|}
\hline Site ID & Site name & $\begin{array}{l}\text { Latitude } \\
\text { (DDMMSS) }\end{array}$ & $\begin{array}{l}\text { Longitude } \\
\text { (DDDMMSS) }\end{array}$ & $\begin{array}{l}\text { Altitude } \\
\text { (feet) }\end{array}$ & $\begin{array}{l}\text { Well } \\
\text { depth } \\
\text { (feet) }\end{array}$ & $\begin{array}{c}\text { Screen } \\
\text { interval } \\
\text { (feet) }\end{array}$ \\
\hline $3.60837 \mathrm{E}+14$ & 4th\&CLARK & 360955 & 1150836 & 2,010 & 22 & '18-22' \\
\hline $3.60832 \mathrm{E}+14$ & BLM & 360559 & 1145827 & 1,590 & 80 & '76-80' \\
\hline $3.60908 \mathrm{E}+14$ & BROADBENT & 360908 & 1151224 & 2,209 & 27 & '22-27' \\
\hline $3.60621 \mathrm{E}+14$ & $\mathrm{C} 27$ & 360621 & 1150559 & 1,938 & 30 & '24-30' \\
\hline $3.60521 \mathrm{E}+14$ & $\mathrm{C} 28$ & 360521 & 1150422 & 1,803 & 19 & '14-19' \\
\hline $3.60605 \mathrm{E}+14$ & $\mathrm{C} 33$ & 360605 & 1150525 & 1,912 & 24 & '19-24' \\
\hline $3.60647 \mathrm{E}+14$ & $\mathrm{C} 49$ & 360647 & 1150440 & 1,780 & 25 & '20-25' \\
\hline $3.61212 \mathrm{E}+14$ & CARI15 & 361212 & 1150659 & 1,910 & 46 & '43-46' \\
\hline $3.6093 \mathrm{E}+14$ & CHAR-8 & 360930 & 1150834 & 2,008 & 25 & '21-25' \\
\hline $3.61425 \mathrm{E}+14$ & CRAI15 & 361425 & 1150619 & 1,919 & 84 & '80-84' \\
\hline $3.60744 \mathrm{E}+14$ & DIT & 360718 & 1150405 & 1,730 & 11 & '7-11' \\
\hline $3.60547 \mathrm{E}+14$ & DORIS1 & 360547 & 1150528 & 1,919 & 29 & '24-29' \\
\hline $3.6064 \mathrm{E}+14$ & EA\&R & 360640 & 1150704 & 1,948 & 20 & '10-15' \\
\hline $3.60535 \mathrm{E}+14$ & HAC\&ANN & 360535 & 1150509 & 1,872 & 18 & '14-18' \\
\hline $3.60537 \mathrm{E}+14$ & HAC\&LAKEW & 360537 & 1150537 & 1,912 & 23 & '17-23' \\
\hline $3.60924 \mathrm{E}+14$ & HUNTPK & 360924 & 1150811 & 1,990 & 15 & '11-15' \\
\hline $3.60924 \mathrm{E}+14$ & HUNTPK2 & 360924 & 1150811 & 1,990 & 17 & '12-17' \\
\hline $3.60852 \mathrm{E}+14$ & HWYSHR1 & 360852 & 1150609 & 1,825 & 25 & '20-25' \\
\hline $3.60838 \mathrm{E}+14$ & I15SAH & 360838 & 1151018 & 2,075 & 24 & '21-24' \\
\hline $3.60735 \mathrm{E}+14$ & I15SPRING & 360735 & 1151052 & 2,120 & 45 & '41-45' \\
\hline $3.61102 \mathrm{E}+14$ & JGAYPK1 & 361102 & 1150836 & 2,010 & 15 & '11-15' \\
\hline $3.61102 \mathrm{E}+14$ & JGAYPK2 & 361102 & 1150836 & 2,010 & 33 & '28-33' \\
\hline $3.61053 \mathrm{E}+14$ & LV-MGOLF & 361053 & 1151158 & 2,190 & 33 & '27-31' \\
\hline $3.61014 \mathrm{E}+14$ & MDB-6 & 361014 & 1151117 & 2,140 & 40 & '20-40' \\
\hline $3.60601 \mathrm{E}+14$ & NELLIS $\dagger$ & 360601 & 1150344 & 1,720 & $500 \dagger$ & $500 \dagger$ \\
\hline $3.60522 \mathrm{E}+14$ & P.P.VISTA & 360522 & 1150721 & 2,010 & 30 & '26-30' \\
\hline $3.60617 \mathrm{E}+14$ & P.PARK1 & 360617 & 1150638 & 1,950 & 24 & '20-24' \\
\hline $3.60617 \mathrm{E}+14$ & P.PARK2 & 360617 & 1150638 & 1,958 & 30 & '25-30' \\
\hline $3.60937 \mathrm{E}+14$ & ROT1 & 360937 & 1151134 & 2,155 & 35 & '30-35' \\
\hline $3.60937 \mathrm{E}+14$ & ROT2 & 360937 & 1151134 & 2,158 & 18 & '14-18' \\
\hline $3.60648 \mathrm{E}+14$ & SNSC & 360648 & 1150849 & 2,030 & 15 & '10-15' \\
\hline $3.60401 \mathrm{E}+14$ & USGSOS & 360401 & 1150823 & 2,086 & 60 & '55-60' \\
\hline $3.60921 \mathrm{E}+14$ & WALLI15 & 360921 & 1150936 & 2,047 & 17 & '13-14' \\
\hline
\end{tabular}

$\dagger$ The NELLIS well is an artesian well

Download Comma Separated Values (table5a.csv 2k) 
Table 5. Chemical data for groundwater samples from the surficial aquifer, Las Vegas, Nev. A, Site locations and well information; B, Field parameters; C, Major ion chemistry; D, Trace constituents (Set A); E, Trace constituents (Set B). (D, degrees; M, minutes; $S$, seconds; $\mathrm{mg} / \mathrm{L}$, milligrams per liter; std, standard deviation; $\mu \mathrm{S} / \mathrm{cm}$, microseimens per centimeter; ${ }^{\circ} \mathrm{C}$, degrees Celsius; meq/L, milliequivalents per liter; $\mu \mathrm{g} / \mathrm{L}$, micrograms per liter; NS, not sampled).-Continued

Table 5B, Field Parameters

\begin{tabular}{|c|c|c|c|c|c|c|c|}
\hline Site ID & Site name & $\begin{array}{c}\text { Collection } \\
\text { date }\end{array}$ & $\begin{array}{l}\text { Depth to } \\
\text { water } \\
\text { (feet) }\end{array}$ & $\begin{array}{c}\text { Field } \\
\text { dissolved } \\
\text { oxygen } \\
\text { (mg/L) }\end{array}$ & $\begin{array}{c}\text { Field } \\
\text { pH } \\
\text { (std units) }\end{array}$ & $\begin{array}{c}\text { Field } \\
\text { specific } \\
\text { conductivity } \\
(\mu \mathrm{S} / \mathrm{cm})\end{array}$ & $\begin{array}{c}\text { Water } \\
\text { temp } \\
\left({ }^{\circ} \mathrm{C}\right)\end{array}$ \\
\hline $3.60837 \mathrm{E}+14$ & $4^{\text {th }} \&$ CLARK & $8 / 21 / 1993$ & 7.7 & 1.7 & 7.02 & 3100 & 23.6 \\
\hline \multirow[t]{2}{*}{$3.60832 \mathrm{E}+14$} & BLM & 4/16/1993 & 11.2 & 5.7 & 7.36 & 9290 & 25.6 \\
\hline & & $8 / 18 / 1993$ & 10.5 & 0.2 & 7.7 & 11200 & 25.2 \\
\hline $3.60908 \mathrm{E}+14$ & BROADBENT & $8 / 24 / 1993$ & 18 & 3.5 & 7.12 & 2900 & 23 \\
\hline $3.60621 \mathrm{E}+14$ & $\mathrm{C} 27$ & $8 / 24 / 1993$ & 17.9 & 0.9 & 7 & 5950 & 22 \\
\hline $3.60521 \mathrm{E}+14$ & $\mathrm{C} 28$ & 8/19/1993 & 7.8 & 0.7 & 7.1 & 5400 & 27.3 \\
\hline $3.60605 \mathrm{E}+14$ & $\mathrm{C} 33$ & $8 / 19 / 1993$ & 18.2 & 2.3 & 7 & 6040 & 24 \\
\hline $3.60647 \mathrm{E}+14$ & C49 & $8 / 21 / 1993$ & 9.5 & 2.7 & 6.99 & 3650 & 23.3 \\
\hline \multirow[t]{2}{*}{$3.61212 \mathrm{E}+14$} & CARI15 & $4 / 14 / 1993$ & 27.6 & 2.5 & 7.33 & 575 & 24.9 \\
\hline & & $8 / 22 / 1993$ & 27.4 & 3.6 & 7.44 & 598 & 23.1 \\
\hline $3.6093 \mathrm{E}+14$ & CHAR-8 & $8 / 22 / 1993$ & 11.9 & NS & 6.89 & 3400 & 23 \\
\hline $3.61425 \mathrm{E}+14$ & CRAI15 & $8 / 20 / 1993$ & 62.6 & 5.9 & 7.6 & 1390 & 25 \\
\hline \multirow[t]{3}{*}{$3.60744 \mathrm{E}+14$} & DIT & $4 / 16 / 1993$ & 5.3 & 2 & 6.98 & 5490 & 16.4 \\
\hline & & $8 / 18 / 1993$ & 8.3 & 2.1 & 6.94 & 5530 & 24.6 \\
\hline & & 9/13/1994 & 8.4 & 2.6 & 6.98 & 5250 & 21.9 \\
\hline $3.60547 \mathrm{E}+14$ & DORIS1 & $8 / 20 / 1993$ & 16.2 & 3.5 & 7.18 & 4650 & 21.9 \\
\hline $3.6064 \mathrm{E}+14$ & EA\&R & $8 / 20 / 1993$ & 9 & 1.6 & 6.88 & 4490 & 21.6 \\
\hline $3.60535 \mathrm{E}+14$ & HAC \& ANN & $8 / 19 / 1993$ & 6.2 & 2.7 & 7.1 & 5570 & 23.9 \\
\hline $3.60537 \mathrm{E}+14$ & HAC \& LAKEW & 8/19/1993 & 10.3 & 3.2 & 7.07 & 5600 & 21.2 \\
\hline \multirow[t]{2}{*}{$3.60924 \mathrm{E}+14$} & HUNTPK & $8 / 22 / 1993$ & 7.1 & 2.9 & 7.48 & 2970 & 22.9 \\
\hline & & 9/14/1994 & 7.1 & 2.7 & 7.14 & 3000 & 20.9 \\
\hline
\end{tabular}


Table 5. Chemical data for groundwater samples from the surficial aquifer, Las Vegas, Nev. A, Site locations and well information; B, Field parameters; C, Major ion chemistry; D, Trace constituents (Set A); E, Trace constituents (Set B). (D, degrees; M, minutes; $S$, seconds; $m g / L$, milligrams per liter; std, standard deviation; $\mu S / \mathrm{cm}$, microseimens per centimeter; ${ }^{\circ} \mathrm{C}$, degrees Celsius; meq/L, milliequivalents per liter; $\mu \mathrm{g} / \mathrm{L}$, micrograms per liter; NS, not sampled).-Continued

Table 5B, Field Parameters

\begin{tabular}{|c|c|c|c|c|c|c|c|}
\hline Site ID & Site name & $\begin{array}{l}\text { Collection } \\
\text { date }\end{array}$ & $\begin{array}{l}\text { Depth to } \\
\text { water } \\
\text { (feet) }\end{array}$ & $\begin{array}{c}\text { Field } \\
\text { dissolved } \\
\text { oxygen } \\
\text { (mg/L) }\end{array}$ & $\begin{array}{c}\text { Field } \\
\text { pH } \\
\text { (std units) }\end{array}$ & $\begin{array}{c}\text { Field } \\
\text { specific } \\
\text { conductivity } \\
(\mu \mathrm{S} / \mathrm{cm})\end{array}$ & $\begin{array}{l}\text { Water } \\
\text { temp } \\
\left({ }^{\circ} \mathrm{C}\right)\end{array}$ \\
\hline $3.60924 \mathrm{E}+14$ & HUNTPK2 & $9 / 14 / 1994$ & 7.2 & 2.6 & 7.17 & 3020 & 20 \\
\hline $3.60852 \mathrm{E}+14$ & HWYSHR1 & $8 / 20 / 1993$ & 6.9 & 0.4 & 7.05 & 5550 & 24.2 \\
\hline \multirow[t]{2}{*}{$3.60838 \mathrm{E}+14$} & I15SAH & $4 / 14 / 1993$ & 6.7 & 1.8 & 7.36 & 2700 & 21.9 \\
\hline & & $8 / 18 / 1993$ & 7 & 3.1 & 7.2 & 2930 & 24.8 \\
\hline \multirow[t]{2}{*}{$3.60735 \mathrm{E}+14$} & I15SPRING & $4 / 13 / 1993$ & 8.8 & 4.1 & 7.01 & 3310 & 23.3 \\
\hline & & 8/18/1993 & 8.7 & 3.9 & 7 & 3470 & 23.4 \\
\hline $3.61102 \mathrm{E}+14$ & JGAYPK1 & $8 / 23 / 1993$ & 9.2 & 0.4 & 7.07 & 2390 & 21.5 \\
\hline $3.61102 \mathrm{E}+14$ & JGAYPK2 & $8 / 23 / 1993$ & 10 & 0.5 & 7.23 & 2160 & 20.8 \\
\hline $3.61053 \mathrm{E}+14$ & LV-MGOLF & $8 / 22 / 1993$ & 9.6 & 3 & 7.28 & 1600 & 20 \\
\hline \multirow[t]{2}{*}{$3.61014 \mathrm{E}+14$} & MDB-6 & $4 / 13 / 1993$ & 8 & 3.8 & 10.8 & 1190 & 20.3 \\
\hline & & 8/23/1993 & 12.5 & NS & 10.2 & 1290 & 21 \\
\hline \multirow[t]{2}{*}{$3.60601 \mathrm{E}+14$} & NELLIS $\dagger$ & 4/16/1993 & $-63.0 \dagger$ & 4.6 & 7.28 & 586 & 24.2 \\
\hline & & 9/13/1994 & $-62.0 \dagger$ & 4.2 & 7.78 & 622 & 24.6 \\
\hline $3.60522 \mathrm{E}+14$ & P.P.VISTA & $8 / 21 / 1993$ & 16.8 & 4.3 & 6.99 & 2540 & 20.6 \\
\hline \multirow[t]{2}{*}{$3.60617 \mathrm{E}+14$} & P.PARK1 & $4 / 12 / 1993$ & 11.9 & NS & 7.06 & 5000 & 18.7 \\
\hline & & $8 / 17 / 1993$ & 13.8 & NS & 7.32 & 4920 & 23 \\
\hline $3.60617 \mathrm{E}+14$ & P.PARK2 & 8/17/1993 & 13.6 & 2 & 7.06 & 4810 & 20.8 \\
\hline $3.60937 \mathrm{E}+14$ & ROT1 & $8 / 17 / 1993$ & 8.1 & 0.2 & 5.8 & 2300 & 18.5 \\
\hline \multirow[t]{2}{*}{$3.60937 \mathrm{E}+14$} & ROT2 & $4 / 15 / 1993$ & 9.5 & 1.2 & 6.74 & 5530 & 16.8 \\
\hline & & $8 / 17 / 1993$ & 8.9 & 0.2 & 6.66 & 5320 & 19.6 \\
\hline $3.60648 \mathrm{E}+14$ & SNSC & $8 / 23 / 1993$ & 8.4 & 1.6 & 6.95 & 4050 & 24.5 \\
\hline $3.60401 \mathrm{E}+14$ & USGSOS & $8 / 16 / 1993$ & 30 & 3.9 & 6.74 & 1830 & 23.2 \\
\hline \multirow[t]{2}{*}{$3.60921 \mathrm{E}+14$} & WALLI15 & $4 / 13 / 1993$ & 7.8 & 1.6 & 6.76 & 4200 & 21 \\
\hline & & $8 / 21 / 1993$ & 8.7 & 2 & 6.85 & 4650 & 22.6 \\
\hline
\end{tabular}

$\dagger$ The NELLIS well is an artesian well

Download Comma Separated Values (table5b.csv 2k) 
Table 5. Chemical data for groundwater samples from the surficial aquifer, Las Vegas, Nev. A, Site locations and well information; B, Field parameters; C, Major ion chemistry; D, Trace constituents (Set A); E, Trace constituents (Set B). (D, degrees; M, minutes; $S$, seconds; $\mathrm{mg} / \mathrm{L}$, milligrams per liter; std, standard deviation; $\mu \mathrm{S} / \mathrm{cm}$, microseimens per centimeter; ${ }^{\circ} \mathrm{C}$, degrees Celsius; meq/L, milliequivalents per liter; $\mu \mathrm{g} / \mathrm{L}$, micrograms per liter; NS, not sampled).-Continued

Table 5C, Major ion chemistry

\begin{tabular}{|c|c|c|c|c|c|c|c|c|c|c|}
\hline Site ID & Site name & $\begin{array}{l}\text { Collection } \\
\text { date }\end{array}$ & $\begin{array}{l}\text { Alkalinity } \\
\text { (meq/L) }\end{array}$ & $\begin{array}{c}\mathrm{Cl} \\
\text { (meq/L) }\end{array}$ & $\begin{array}{c}\mathrm{SO}_{4} \\
\text { (meq/L) }\end{array}$ & $\begin{array}{c}\mathrm{Ca} \\
\text { (meq/L) }\end{array}$ & $\begin{array}{c}\mathrm{Mg} \\
\text { (meq/L) }\end{array}$ & $\begin{array}{c}\mathrm{Na} \\
\text { (meq/L) }\end{array}$ & $\begin{array}{c}\mathrm{K} \\
\text { (meq/L) }\end{array}$ & $\begin{array}{c}\text { lon } \\
\text { balance } \\
(\%)\end{array}$ \\
\hline $3.6084 \mathrm{E}+14$ & $4^{\text {th }} \&$ CLARK & $8 / 21 / 1993$ & 6.24 & 5.2 & 29 & 10.7 & 21.4 & 6.89 & 0.83 & -0.77 \\
\hline \multirow[t]{2}{*}{$3.6083 \mathrm{E}+14$} & BLM & 4/16/1993 & 1.33 & 36.3 & 73 & 26.7 & 42.7 & 49.3 & 2.8 & 4.68 \\
\hline & & $8 / 18 / 1993$ & 1.79 & 50.4 & 94 & 26.6 & 47.2 & 56.1 & 2.23 & -5.05 \\
\hline $3.6091 \mathrm{E}+14$ & BROADBENT & $8 / 24 / 1993$ & 5.06 & 5.4 & 27 & 12.9 & 17.5 & 7.36 & 0.33 & 0.83 \\
\hline $3.6062 \mathrm{E}+14$ & $\mathrm{C} 27$ & $8 / 24 / 1993$ & 4.19 & 7.2 & 78 & 24.8 & 43.5 & 18.1 & 0.94 & -1.16 \\
\hline $3.6052 \mathrm{E}+14$ & $\mathrm{C} 28$ & $8 / 19 / 1993$ & 4.61 & 28.6 & 48 & 22.9 & 22.3 & 19.9 & 1.16 & -10.14 \\
\hline $3.6061 \mathrm{E}+14$ & $\mathrm{C} 33$ & $8 / 19 / 1993$ & 4.01 & 13.6 & 73 & 23.4 & 33.2 & 17.6 & 1.44 & -9 \\
\hline $3.6065 \mathrm{E}+14$ & C49 & $8 / 21 / 1993$ & 4.17 & 8.8 & 37 & 15.4 & 15.5 & 10.1 & 0.79 & -8.91 \\
\hline \multirow[t]{2}{*}{$3.6121 \mathrm{E}+14$} & CARI15 & $4 / 14 / 1993$ & 4.66 & 0.3 & $<2$ & 3.35 & 3.01 & 0.37 & 0.06 & 15.57 \\
\hline & & $8 / 22 / 1993$ & 4.81 & 0.4 & $<2$ & 3.19 & 2.85 & 0.346 & 0.08 & 10.76 \\
\hline $3.6093 \mathrm{E}+14$ & CHAR-8 & $8 / 22 / 1993$ & 5.67 & 5.7 & 35 & 17.1 & 24.8 & 7.11 & 0.69 & 3.47 \\
\hline $3.6143 \mathrm{E}+14$ & CRAI15 & $8 / 20 / 1993$ & 3.67 & 4.8 & 7 & 3.71 & 9.92 & 0.566 & 0.09 & -3.98 \\
\hline \multirow[t]{3}{*}{$3.6074 \mathrm{E}+14$} & DIT & $4 / 16 / 1993$ & 5.18 & 14.1 & 48 & 25.6 & 28.2 & 17.8 & 1.71 & 4.29 \\
\hline & & $8 / 18 / 1993$ & 5.35 & 14.8 & 58 & 23.8 & 26.9 & 14.5 & 1.86 & -7.64 \\
\hline & & 9/13/1994 & 5.31 & 13.5 & 45 & 23.9 & 26.9 & 19 & 1.55 & 5.58 \\
\hline $3.6055 \mathrm{E}+14$ & DORIS1 & $8 / 20 / 1993$ & 3.06 & 10.4 & 49 & 21.6 & 28.1 & 9.05 & 0.92 & -2.28 \\
\hline $3.6064 \mathrm{E}+14$ & EA\&R & $8 / 20 / 1993$ & 4.84 & 7.9 & 45 & 26.7 & 21.6 & 14.5 & 0.77 & 4.81 \\
\hline $3.6054 \mathrm{E}+14$ & HAC\&ANN & $8 / 19 / 1993$ & 3.68 & 14.5 & 62 & 26.9 & 29.2 & 18.2 & 0.85 & -3.24 \\
\hline $3.6054 \mathrm{E}+14$ & HAC\&LAKEW & $8 / 19 / 1993$ & 3.43 & 14.7 & 58 & 26.7 & 33 & 16.6 & 1.14 & 0.85 \\
\hline \multirow[t]{2}{*}{$3.6092 \mathrm{E}+14$} & HUNTPK & $8 / 22 / 1993$ & 4.24 & 5.8 & 30 & 11.4 & 19.4 & 6.49 & 0.54 & -2.84 \\
\hline & & 9/14/1994 & 4.05 & 5.3 & 25 & 12.4 & 20.9 & 7.74 & 0.41 & 9.37 \\
\hline $3.6092 \mathrm{E}+14$ & HUNTPK2 & 9/14/1994 & 3.87 & 5.5 & 26 & 12.2 & 20.4 & 7.61 & 0.41 & 6.91 \\
\hline
\end{tabular}


Table 5. Chemical data for groundwater samples from the surficial aquifer, Las Vegas, Nev. A, Site locations and well information; B, Field parameters; C, Major ion chemistry; D, Trace constituents (Set A); E, Trace constituents (Set $B$ ). (D, degrees; M, minutes; $S$, seconds; mg/L, milligrams per liter; std, standard deviation; $\mu S / \mathrm{cm}$, microseimens per centimeter; ${ }^{\circ} \mathrm{C}$, degrees Celsius; meq/L, milliequivalents per liter; $\mu \mathrm{g} / \mathrm{L}$, micrograms per liter; NS, not sampled).-Continued

Table 5C, Major ion chemistry

\begin{tabular}{|c|c|c|c|c|c|c|c|c|c|c|}
\hline Site ID & Site name & $\begin{array}{l}\text { Collection } \\
\text { date }\end{array}$ & $\begin{array}{c}\text { Alkalinity } \\
\text { (meq/L) }\end{array}$ & $\begin{array}{c}\mathrm{Cl} \\
(\mathrm{meq} / \mathrm{L})\end{array}$ & $\begin{array}{c}\mathrm{SO}_{4} \\
\text { (meq/L) }\end{array}$ & $\begin{array}{c}\mathrm{Ca} \\
(\mathrm{meq} / \mathrm{L})\end{array}$ & $\begin{array}{c}\mathrm{Mg} \\
(\mathrm{meq} / \mathrm{L})\end{array}$ & $\begin{array}{c}\mathrm{Na} \\
\text { (meq/L) }\end{array}$ & $\underset{(\mathrm{meq} / \mathrm{L})}{\mathrm{K}}$ & $\begin{array}{c}\text { Ion } \\
\text { balance } \\
(\%)\end{array}$ \\
\hline $3.6085 \mathrm{E}+14$ & HWYSHR1 & $8 / 20 / 1993$ & 4.67 & 12.1 & 52 & 21.8 & 27.7 & 22.7 & 1.33 & 3.35 \\
\hline \multirow[t]{2}{*}{$3.6084 \mathrm{E}+14$} & I15SAH & 4/14/1993 & 3.67 & 4.5 & 19 & 13.4 & 16.3 & 7.36 & 0.75 & 16.37 \\
\hline & & $8 / 18 / 1993$ & 3.9 & 6.4 & 27 & 11.4 & 14.7 & 6.13 & 0.9 & -5.92 \\
\hline \multirow[t]{2}{*}{$3.6074 \mathrm{E}+14$} & I15SPRING & $4 / 13 / 1993$ & 3.87 & 6.3 & 28 & 16.2 & 18.9 & 8.65 & 0.45 & 7.32 \\
\hline & & $8 / 18 / 1993$ & 3.95 & 7 & 34 & 17.4 & 18.4 & 10.3 & 0.42 & 1.72 \\
\hline $3.611 \mathrm{E}+14$ & JGAYPK1 & $8 / 23 / 1993$ & 6.11 & 4.7 & 15 & 7.99 & 11.6 & 4.98 & 0.81 & -0.84 \\
\hline $3.611 \mathrm{E}+14$ & JGAYPK2 & $8 / 23 / 1993$ & 5.04 & 4.2 & 15 & 5.19 & 11.3 & 4.48 & 0.52 & -6.01 \\
\hline $3.6105 \mathrm{E}+14$ & LV-MGOLF & $8 / 22 / 1993$ & 4.35 & 3.8 & 9 & 8.47 & 8.94 & 2.16 & 0.14 & 6.95 \\
\hline \multirow[t]{2}{*}{$3.6101 \mathrm{E}+14$} & MDB-6 & $4 / 13 / 1993$ & 0.89 & 1.7 & 5 & 8.66 & 0.317 & 2.94 & 0.3 & 23.36 \\
\hline & & $8 / 23 / 1993$ & 0.833 & 2.8 & 10 & 9.4 & 0.0295 & 1.99 & 0.34 & -7.38 \\
\hline \multirow[t]{2}{*}{$3.606 \mathrm{E}+14$} & NELLIS & $4 / 16 / 1993$ & 3.05 & 0.2 & 3 & 1.88 & 1.99 & 2.61 & 0.17 & 3.1 \\
\hline & & 9/13/1994 & 3.05 & 0.4 & 3 & 1.99 & 2.07 & 2.77 & 0.23 & 4.52 \\
\hline $3.6052 \mathrm{E}+14$ & P.P.VISTA & $8 / 21 / 1993$ & 3.76 & 7.4 & 35 & 20.4 & 17.1 & 11.1 & 0.49 & 3.08 \\
\hline \multirow[t]{2}{*}{$3.6062 \mathrm{E}+14$} & P.PARK1 & $4 / 12 / 1993$ & 3.85 & 7.8 & 50 & 22.9 & 28.3 & 17.5 & 0.74 & 5.94 \\
\hline & & $8 / 17 / 1993$ & 3.86 & 8.2 & 58 & 23.6 & 27.4 & 17.6 & 0.61 & -0.61 \\
\hline $3.6062 \mathrm{E}+14$ & P.PARK2 & 8/17/1993 & 3.82 & 6.7 & 50 & 23.3 & 25.9 & 17.9 & 0.63 & 5.62 \\
\hline $3.6094 \mathrm{E}+14$ & ROT1 & $8 / 17 / 1993$ & 7.68 & 3.5 & 17 & 9.2 & 13.3 & 6.23 & 0.21 & 1.33 \\
\hline \multirow[t]{2}{*}{$3.6094 \mathrm{E}+14$} & ROT2 & $4 / 15 / 1993$ & 8.82 & 13.8 & 44 & 18.1 & 38.8 & 20.7 & 1.12 & 8.33 \\
\hline & & 8/17/1993 & 8.88 & 13.1 & 43 & 16.3 & 34.7 & 16.3 & 0.88 & 2.4 \\
\hline $3.6065 E+14$ & SNSC & $8 / 23 / 1993$ & 5.03 & 6.4 & 44 & 25.1 & 21.3 & 11.4 & 0.81 & 2.79 \\
\hline $3.604 \mathrm{E}+14$ & USGSOS & $8 / 16 / 1993$ & 3.16 & 2.1 & 16 & 12.2 & 8.61 & 2.26 & 0.23 & 4.58 \\
\hline \multirow[t]{2}{*}{$3.6092 \mathrm{E}+14$} & WALLI15 & 4/13/1993 & 6.21 & 6.7 & 46 & 21.8 & 34.5 & 10.2 & 1.05 & 6.83 \\
\hline & & $8 / 21 / 1993$ & 6.07 & 6.7 & 50 & 22.4 & 33.6 & 9.24 & 1.14 & 2.8 \\
\hline
\end{tabular}

Download Comma Separated Values (table5c.csv3k) 
Table 5. Chemical data for groundwater samples from the surficial aquifer, Las Vegas, Nev. $A$, Site locations and well information; B, Field parameters; C, Major ion chemistry; D, Trace constituents (Set A); E, Trace constituents (Set $B)$. (D, degrees; M, minutes; $S$, seconds; mg/L, milligrams per liter; std, standard deviation; $\mu S / \mathrm{cm}$, microseimens per centimeter; ${ }^{\circ} \mathrm{C}$, degrees Celsius; meq/L, milliequivalents per liter; $\mu \mathrm{g} / \mathrm{L}$, micrograms per liter; NS, not sampled).-Continued

Table 5D, Trace constituents (Set A)

\begin{tabular}{|c|c|c|c|c|c|c|c|c|c|c|}
\hline Site ID & Site name & $\begin{array}{l}\text { Collection } \\
\text { date }\end{array}$ & $\begin{array}{c}\text { DOC } \\
\text { (mg/L) }\end{array}$ & $\begin{array}{c}\text { B } \\
(\mathrm{mg} / \mathrm{L})\end{array}$ & $\begin{array}{c}\mathrm{Ba} \\
(\mathrm{mg} / \mathrm{L})\end{array}$ & $\begin{array}{c}\mathrm{Be} \\
(\mathrm{mg} / \mathrm{L})\end{array}$ & $\begin{array}{c}\mathrm{Cd} \\
(\mathrm{mg} / \mathrm{L})\end{array}$ & $\begin{array}{c}\text { Co } \\
\text { (mg/L) }\end{array}$ & $\begin{array}{c}\mathrm{Cu} \\
(\mathrm{mg} / \mathrm{L})\end{array}$ & $\begin{array}{c}\mathrm{Fe} \\
(\mathrm{mg} / \mathrm{L})\end{array}$ \\
\hline $3.60837 \mathrm{E}+14$ & $4^{\text {th }} \&$ CLARK & $8 / 21 / 1993$ & 2 & 0.523 & 0.012 & $<0.001$ & $<0.01$ & $<0.01$ & $<0.005$ & $<0.005$ \\
\hline \multirow[t]{2}{*}{$3.60832 \mathrm{E}+14$} & BLM & $4 / 16 / 1993$ & 1.9 & 4.21 & .011 & .002 & $<.01$ & $<.01$ & .006 & $<.005$ \\
\hline & & $8 / 18 / 1993$ & 1.8 & 4.38 & .01 & $<.001$ & $<.01$ & $<.01$ & $<.005$ & $<.005$ \\
\hline $3.60908 \mathrm{E}+14$ & BROADBENT & $8 / 24 / 1993$ & 3.1 & .936 & .017 & $<.001$ & $<.01$ & $<.01$ & $<.005$ & .047 \\
\hline $3.60621 \mathrm{E}+14$ & $\mathrm{C} 27$ & $8 / 24 / 1993$ & 2.7 & 1.82 & .007 & $<.001$ & $<.01$ & $<.01$ & $<.005$ & .012 \\
\hline $3.60521 \mathrm{E}+14$ & $\mathrm{C} 28$ & 8/19/1993 & 1 & 1.29 & .014 & $<.001$ & $<.01$ & $<.01$ & $<.005$ & .012 \\
\hline $3.60605 \mathrm{E}+14$ & $\mathrm{C} 33$ & $8 / 19 / 1993$ & 3.7 & 3.61 & .012 & $<.001$ & $<.01$ & $<.01$ & $<.005$ & $<.005$ \\
\hline $3.60647 \mathrm{E}+14$ & $\mathrm{C} 49$ & $8 / 21 / 1993$ & 1.7 & 1.25 & .012 & $<.001$ & $<.01$ & $<.01$ & $<.005$ & $<.005$ \\
\hline \multirow[t]{2}{*}{$3.61212 \mathrm{E}+14$} & CARI15 & $4 / 14 / 1993$ & 1.5 & .046 & .201 & $<.001$ & $<.01$ & $<.01$ & $<.005$ & .34 \\
\hline & & $8 / 22 / 1993$ & .6 & .035 & .181 & .002 & $<.01$ & $<.01$ & $<.005$ & $<.005$ \\
\hline $3.6093 \mathrm{E}+14$ & CHAR-8 & $8 / 22 / 1993$ & 2.9 & .518 & .013 & $<.001$ & $<.01$ & $<.01$ & $<.005$ & $<.005$ \\
\hline $3.61425 \mathrm{E}+14$ & CRAI15 & $8 / 20 / 1993$ & 5.8 & .032 & .178 & $<.001$ & $<.01$ & $<.01$ & $<.005$ & $<.005$ \\
\hline \multirow[t]{3}{*}{$3.60744 \mathrm{E}+14$} & DIT & $4 / 16 / 1993$ & 3.7 & 2.02 & .016 & $<.001$ & $<.01$ & $<.01$ & $<.005$ & .013 \\
\hline & & $8 / 18 / 1993$ & 3.4 & 1.51 & .01 & $<.001$ & $<.01$ & $<.01$ & $<.005$ & .035 \\
\hline & & 9/13/1994 & 3.2 & 4.02 & .014 & $<.001$ & $<.01$ & $<.01$ & $<.005$ & .07 \\
\hline $3.60547 \mathrm{E}+14$ & DORIS1 & $8 / 20 / 1993$ & 1.8 & 2.55 & .008 & $<.001$ & $<.01$ & $<.01$ & $<.005$ & $<.005$ \\
\hline $3.6064 \mathrm{E}+14$ & EA\&R & $8 / 20 / 1993$ & 3.9 & 1.55 & .01 & $<.001$ & $<.01$ & $<.01$ & $<.005$ & $<.005$ \\
\hline $3.60535 \mathrm{E}+14$ & HAC\&ANN & 8/19/1993 & 1.7 & 2.3 & .008 & $<.001$ & $<.01$ & $<.01$ & $<.005$ & $<.005$ \\
\hline $3.60537 \mathrm{E}+14$ & HAC\&LAKEW & $8 / 19 / 1993$ & 1.5 & 1.95 & .008 & $<.001$ & $<.01$ & $<.01$ & $<.005$ & $<.005$ \\
\hline \multirow[t]{2}{*}{$3.60924 \mathrm{E}+14$} & HUNTPK & $8 / 22 / 1993$ & 20 & .357 & .023 & $<.001$ & $<.01$ & $<.01$ & $<.005$ & .082 \\
\hline & & $9 / 14 / 1994$ & 1.45 & .993 & .028 & $<.001$ & $<.01$ & $<.01$ & $<.005$ & $<.005$ \\
\hline
\end{tabular}


Table 5. Chemical data for groundwater samples from the surficial aquifer, Las Vegas, Nev. A, Site locations and well information; B, Field parameters; C, Major ion chemistry; D, Trace constituents (Set A); E, Trace constituents (Set B). (D, degrees; M, minutes; $S$, seconds; $\mathrm{mg} / \mathrm{L}$, milligrams per liter; std, standard deviation; $\mu \mathrm{S} / \mathrm{cm}$, microseimens per centimeter; ${ }^{\circ} \mathrm{C}$, degrees Celsius; meq/L, milliequivalents per liter; $\mu \mathrm{g} / \mathrm{L}$, micrograms per liter; NS, not sampled).-Continued

Table 5D, Trace constituents (Set A)

\begin{tabular}{|c|c|c|c|c|c|c|c|c|c|c|}
\hline Site ID & Site name & $\begin{array}{c}\text { Collection } \\
\text { date }\end{array}$ & $\begin{array}{c}\text { DOC } \\
(\mathrm{mg} / \mathrm{L})\end{array}$ & $\begin{array}{c}B \\
(\mathrm{mg} / \mathrm{L})\end{array}$ & $\begin{array}{c}\mathrm{Ba} \\
(\mathrm{mg} / \mathrm{L})\end{array}$ & $\begin{array}{c}\mathrm{Be} \\
(\mathrm{mg} / \mathrm{L})\end{array}$ & $\begin{array}{c}\mathrm{Cd} \\
(\mathrm{mg} / \mathrm{L})\end{array}$ & $\begin{array}{c}\text { Co } \\
\text { (mg/L) }\end{array}$ & $\begin{array}{c}\mathrm{Cu} \\
(\mathrm{mg} / \mathrm{L})\end{array}$ & $\begin{array}{c}\mathrm{Fe} \\
(\mathrm{mg} / \mathrm{L})\end{array}$ \\
\hline $3.60924 \mathrm{E}+14$ & HUNTPK2 & $9 / 14 / 1994$ & 1.3 & .919 & .017 & $<.001$ & $<.01$ & $<.01$ & $<.005$ & $<.005$ \\
\hline $3.60852 \mathrm{E}+14$ & HWYSHR1 & $8 / 20 / 1993$ & 3.1 & 1.96 & .027 & $<.001$ & $<.01$ & $<.01$ & $<.005$ & .529 \\
\hline \multirow[t]{2}{*}{$3.60838 \mathrm{E}+14$} & I15SAH & 4/14/1993 & 3.1 & .95 & .038 & $<.001$ & $<.01$ & $<.01$ & $<.005$ & .863 \\
\hline & & 8/18/1993 & 1.9 & .783 & .017 & $<.001$ & $<.01$ & $<.01$ & $<.005$ & $<.005$ \\
\hline \multirow[t]{2}{*}{$3.60735 \mathrm{E}+14$} & I15SPRING & 4/13/1993 & 1.9 & 1.22 & .014 & $<.001$ & $<.01$ & $<.01$ & $<.005$ & $<.005$ \\
\hline & & $8 / 18 / 1993$ & 1.6 & .711 & .009 & $<.001$ & $<.01$ & $<.01$ & $<.005$ & $<.005$ \\
\hline $3.61102 \mathrm{E}+14$ & JGAYPK1 & $8 / 23 / 1993$ & 2.3 & .714 & .031 & $<.001$ & $<.01$ & $<.01$ & $<.005$ & $<.005$ \\
\hline $3.61102 \mathrm{E}+14$ & JGAYPK2 & $8 / 23 / 1993$ & 1.5 & .597 & .017 & $<.001$ & $<.01$ & $<.01$ & $<.005$ & $<.005$ \\
\hline $3.61053 \mathrm{E}+14$ & LV-MGOLF & $8 / 22 / 1993$ & 1.6 & .094 & .043 & $<.001$ & $<.01$ & $<.01$ & $<.005$ & $<.005$ \\
\hline \multirow[t]{2}{*}{$3.61014 \mathrm{E}+14$} & MDB-6 & $4 / 13 / 1993$ & 1.7 & .2 & .047 & $<.001$ & $<.01$ & $<.01$ & .016 & $<.005$ \\
\hline & & $8 / 23 / 1993$ & 1.2 & .202 & .039 & $<.001$ & $<.01$ & $<.01$ & $<.005$ & $<.005$ \\
\hline \multirow[t]{2}{*}{$3.60601 \mathrm{E}+14$} & NELLIS & $4 / 16 / 1993$ & .5 & .258 & .032 & $<.001$ & $<.01$ & $<.01$ & $<.005$ & .046 \\
\hline & & 9/13/1994 & .6 & .501 & .041 & $<.001$ & $<.01$ & $<.01$ & $<.005$ & $<.005$ \\
\hline $3.60522 \mathrm{E}+14$ & P.P.VISTA & $8 / 21 / 1993$ & 2.3 & .707 & .016 & $<.001$ & $<.01$ & $<.01$ & $<.005$ & .035 \\
\hline \multirow[t]{2}{*}{$3.60617 \mathrm{E}+14$} & P.PARK1 & $4 / 12 / 1993$ & 3.1 & 2.19 & .011 & $<.001$ & $<.01$ & $<.01$ & $<.005$ & .02 \\
\hline & & 8/17/1993 & 2.7 & 1.79 & .008 & $<.001$ & $<.01$ & $<.01$ & $<.005$ & $<.005$ \\
\hline $3.60617 \mathrm{E}+14$ & P.PARK2 & $8 / 17 / 1993$ & 2.1 & 1.97 & .011 & .001 & $<.01$ & $<.01$ & $<.005$ & $<.005$ \\
\hline $3.60937 \mathrm{E}+14$ & ROT1 & 8/17/1993 & 2.5 & .75 & .017 & $<.001$ & $<.01$ & $<.01$ & $<.005$ & .094 \\
\hline \multirow[t]{2}{*}{$3.60937 \mathrm{E}+14$} & ROT2 & $4 / 15 / 1993$ & 16 & .631 & .036 & .002 & $<.01$ & $<.01$ & .015 & .007 \\
\hline & & $8 / 17 / 1993$ & 14.6 & .463 & .028 & $<.001$ & $<.01$ & $<.01$ & $<.005$ & .035 \\
\hline $3.60648 \mathrm{E}+14$ & SNSC & $8 / 23 / 1993$ & 4.3 & 1.2 & .014 & $<.001$ & $<.01$ & $<.01$ & $<.005$ & $<.005$ \\
\hline $3.60401 \mathrm{E}+14$ & USGSOS & 8/16/1993 & .5 & .196 & .01 & $<.001$ & $<.01$ & $<.01$ & $<.005$ & $<.005$ \\
\hline \multirow[t]{2}{*}{$3.60921 \mathrm{E}+14$} & WALLI15 & $4 / 13 / 1993$ & 3.4 & 1.91 & .022 & $<.001$ & $<.01$ & $<.01$ & $<.005$ & $<.005$ \\
\hline & & $8 / 21 / 1993$ & 3.5 & 1.67 & .021 & $<.001$ & $<.01$ & $<.01$ & $<.005$ & $<.005$ \\
\hline
\end{tabular}

Download Comma Separated Values (table5d.csv 3k) 
Table 5. Chemical data for groundwater samples from the surficial aquifer, Las Vegas, Nev. A, Site locations and well information; B, Field parameters; C, Major ion chemistry; D, Trace constituents (Set A); E, Trace constituents (Set $B)$. (D, degrees; $M$, minutes; $S$, seconds; mg/L, milligrams per liter; std, standard deviation; $\mu S / \mathrm{cm}$, microseimens per centimeter; ${ }^{\circ} \mathrm{C}$, degrees Celsius; meq/L, milliequivalents per liter; $\mu g / L$, micrograms per liter; NS, not sampled).-Continued

Table 5E, Trace constituents (Set B)

\begin{tabular}{|c|c|c|c|c|c|c|c|c|c|c|}
\hline Site ID & Site name & $\begin{array}{c}\text { Collection } \\
\text { date }\end{array}$ & $\begin{array}{c}\mathrm{Li} \\
(\mathrm{mg} / \mathrm{L})\end{array}$ & $\begin{array}{c}\mathrm{Mn} \\
\text { (mg/L) }\end{array}$ & $\begin{array}{c}\mathrm{SiO}_{2} \\
\text { (mg/L) }\end{array}$ & $\begin{array}{c}\mathrm{Sr} \\
(\mathrm{mg} / \mathrm{L})\end{array}$ & $\begin{array}{c}V \\
(\mathrm{mg} / \mathrm{L})\end{array}$ & $\begin{array}{c}\mathrm{Zn} \\
(\mathrm{mg} / \mathrm{L})\end{array}$ & $\begin{array}{c}\text { As } \\
(\mu \mathrm{g} / \mathrm{L})\end{array}$ & $\begin{array}{c}\mathrm{Se} \\
(\mu \mathrm{g} / \mathrm{L})\end{array}$ \\
\hline $3.60837 \mathrm{E}+14$ & $4^{\text {th }} \&$ CLARK & $8 / 21 / 1993$ & 0.105 & 0.003 & 42.3 & 5.64 & $<0.005$ & $<0.01$ & NS & NS \\
\hline \multirow[t]{2}{*}{$3.60832 \mathrm{E}+14$} & BLM & 4/16/1993 & 1.11 & .008 & 19.1 & 12.4 & $<.005$ & $<.01$ & 14 & 34 \\
\hline & & 8/18/1993 & .578 & .006 & 11.5 & 10.1 & .032 & .23 & 35 & 44 \\
\hline $3.60908 \mathrm{E}+14$ & BROADBENT & $8 / 24 / 1993$ & .051 & .011 & 18.5 & 2.12 & $<.005$ & $<.01$ & NS & NS \\
\hline $3.60621 \mathrm{E}+14$ & $\mathrm{C} 27$ & $8 / 24 / 1993$ & .183 & .004 & 21.9 & 5.56 & $<.005$ & $<.01$ & NS & NS \\
\hline $3.60521 \mathrm{E}+14$ & $\mathrm{C} 28$ & 8/19/1993 & .374 & .006 & 46.1 & 7.01 & $<.005$ & .03 & NS & NS \\
\hline $3.60605 \mathrm{E}+14$ & $\mathrm{C} 33$ & 8/19/1993 & .279 & .006 & 25.2 & 7.15 & $<.005$ & $<.01$ & NS & NS \\
\hline $3.60647 \mathrm{E}+14$ & C49 & $8 / 21 / 1993$ & .215 & $<.002$ & 34.3 & 7.55 & $<.005$ & $<.01$ & NS & NS \\
\hline \multirow[t]{2}{*}{$3.61212 \mathrm{E}+14$} & CARI15 & $4 / 14 / 1993$ & .014 & .038 & 25.5 & .794 & $<.005$ & $<.01$ & 2 & 1 \\
\hline & & $8 / 22 / 1993$ & .012 & .004 & 21.3 & .708 & $<.005$ & $<.01$ & 2 & 2 \\
\hline $3.6093 \mathrm{E}+14$ & CHAR-8 & $8 / 22 / 1993$ & .047 & .041 & 28.6 & 5.36 & $<.005$ & .02 & NS & NS \\
\hline $3.61425 \mathrm{E}+14$ & CRAI15 & $8 / 20 / 1993$ & .018 & .019 & 47.5 & 2.17 & .007 & $<.01$ & NS & NS \\
\hline \multirow[t]{3}{*}{$3.60744 \mathrm{E}+14$} & DIT & 4/16/1993 & .234 & .039 & 56.4 & 11.6 & $<.005$ & $<.01$ & NS & NS \\
\hline & & 8/18/1993 & .137 & .008 & 43.1 & 8.91 & .007 & $<.01$ & NS & NS \\
\hline & & 9/13/1994 & .248 & .047 & 66.7 & 11 & $<.005$ & $<.01$ & NS & NS \\
\hline $3.60547 \mathrm{E}+14$ & DORIS1 & $8 / 20 / 1993$ & .163 & .003 & 23.9 & 5.99 & $<.005$ & $<.01$ & NS & NS \\
\hline $3.6064 \mathrm{E}+14$ & EA\&R & $8 / 20 / 1993$ & .193 & .002 & 61.9 & 9 & $<.005$ & $<.01$ & NS & NS \\
\hline $3.60535 \mathrm{E}+14$ & HAC\&ANN & 8/19/1993 & .187 & .005 & 31.3 & 7.3 & $<.005$ & $<.01$ & NS & NS \\
\hline $3.60537 \mathrm{E}+14$ & HAC\&LAKEW & 8/19/1993 & .178 & $<.002$ & 34.6 & 6.82 & $<.005$ & $<.01$ & NS & NS \\
\hline
\end{tabular}


Table 5. Chemical data for groundwater samples from the surficial aquifer, Las Vegas, Nev. A, Site locations and well information; B, Field parameters; C, Major ion chemistry; D, Trace constituents (Set A); E, Trace constituents (Set B). (D, degrees; M, minutes; $S$, seconds; $\mathrm{mg} / \mathrm{L}$, milligrams per liter; std, standard deviation; $\mu \mathrm{S} / \mathrm{cm}$, microseimens per centimeter; ${ }^{\circ} \mathrm{C}$, degrees Celsius; meq/L, milliequivalents per liter; $\mu \mathrm{g} / \mathrm{L}$, micrograms per liter; NS, not sampled).-Continued

Table 5E, Trace constituents (Set B)

\begin{tabular}{|c|c|c|c|c|c|c|c|c|c|c|}
\hline Site ID & Site name & $\begin{array}{c}\text { Collection } \\
\text { date }\end{array}$ & $\begin{array}{c}\mathrm{Li} \\
(\mathrm{mg} / \mathrm{L}) \\
\end{array}$ & $\begin{array}{c}M n \\
(\mathrm{mg} / \mathrm{L}) \\
\end{array}$ & $\begin{array}{c}\mathrm{SiO}_{2} \\
(\mathrm{mg} / \mathrm{L})\end{array}$ & $\begin{array}{c}\mathrm{Sr} \\
(\mathrm{mg} / \mathrm{L})\end{array}$ & $\begin{array}{c}V \\
(\mathrm{mg} / \mathrm{L})\end{array}$ & $\begin{array}{c}\mathrm{Zn} \\
(\mathrm{mg} / \mathrm{L})\end{array}$ & $\begin{array}{c}\text { As } \\
(\mu \mathrm{g} / \mathrm{L})\end{array}$ & $\begin{array}{c}\text { Se } \\
(\mu g / L)\end{array}$ \\
\hline \multirow[t]{2}{*}{$3.60924 \mathrm{E}+14$} & HUNTPK & $8 / 22 / 1993$ & .037 & .007 & 17.5 & 2.51 & $<.005$ & .01 & NS & NS \\
\hline & & 9/14/1994 & .064 & $<.002$ & 27 & 3.15 & $<.005$ & .13 & NS & NS \\
\hline $3.60924 \mathrm{E}+14$ & HUNTPK2 & $9 / 14 / 1994$ & .064 & $<.002$ & 27.5 & 3.13 & $<.005$ & $<.01$ & NS & NS \\
\hline $3.60852 \mathrm{E}+14$ & HWYSHR1 & $8 / 20 / 1993$ & .149 & .095 & 55.5 & 6.12 & $<.005$ & .04 & NS & NS \\
\hline \multirow[t]{2}{*}{$3.60838 \mathrm{E}+14$} & I15SAH & 4/14/1993 & .075 & .039 & 28.7 & 3.2 & $<.005$ & $<.01$ & NS & NS \\
\hline & & $8 / 18 / 1993$ & .067 & $<.002$ & 20.8 & 2.84 & $<.005$ & $<.01$ & NS & NS \\
\hline \multirow[t]{2}{*}{$3.60735 \mathrm{E}+14$} & I15SPRING & $4 / 13 / 1993$ & .076 & $<.002$ & 22 & 4.52 & $<.005$ & .03 & NS & NS \\
\hline & & $8 / 18 / 1993$ & .038 & $<.002$ & 14.2 & 3.1 & $<.005$ & $<.01$ & NS & NS \\
\hline $3.61102 \mathrm{E}+14$ & JGAYPK1 & $8 / 23 / 1993$ & .052 & .121 & 40.4 & 5.05 & $<.005$ & $<.01$ & NS & NS \\
\hline $3.61102 \mathrm{E}+14$ & JGAYPK2 & $8 / 23 / 1993$ & .029 & .01 & 23.8 & 2.23 & $<.005$ & .02 & NS & NS \\
\hline $3.61053 \mathrm{E}+14$ & LV-MGOLF & $8 / 22 / 1993$ & .013 & $<.002$ & 16.9 & 1.21 & $<.005$ & $<.01$ & 2 & 12 \\
\hline \multirow[t]{2}{*}{$3.61014 \mathrm{E}+14$} & MDB-6 & 4/13/1993 & .025 & $<.002$ & 14.1 & 1.24 & .01 & $<.01$ & NS & NS \\
\hline & & $8 / 23 / 1993$ & .018 & $<.002$ & 14.3 & 1.14 & .007 & $<.01$ & NS & NS \\
\hline \multirow[t]{2}{*}{$3.60601 \mathrm{E}+14$} & NELLIS & 4/16/1993 & .137 & $<.002$ & 21.1 & .788 & .009 & $<.01$ & 10 & 1 \\
\hline & & 9/13/1994 & .12 & $<.002$ & 23.3 & .793 & $<.005$ & .12 & NS & NS \\
\hline $3.60522 \mathrm{E}+14$ & P.P.VISTA & $8 / 21 / 1993$ & .061 & .118 & 17.9 & 4.44 & $<.005$ & .02 & NS & NS \\
\hline \multirow[t]{2}{*}{$3.60617 \mathrm{E}+14$} & P.PARK1 & 4/12/1993 & .187 & .004 & 31.2 & 7.61 & $<.005$ & $<.01$ & 10 & 33 \\
\hline & & $8 / 17 / 1993$ & .162 & .004 & 28.4 & 6.49 & $<.005$ & $<.01$ & 10 & 34 \\
\hline $3.60617 \mathrm{E}+14$ & P.PARK2 & 8/17/1993 & .172 & .008 & 31.2 & 7.11 & $<.005$ & $<.01$ & NS & NS \\
\hline $3.60937 \mathrm{E}+14$ & ROT1 & 8/17/1993 & .024 & .015 & 20.5 & 1.41 & $<.005$ & .02 & NS & NS \\
\hline \multirow[t]{2}{*}{$3.60937 \mathrm{E}+14$} & ROT2 & $4 / 15 / 1993$ & .104 & .234 & 31.1 & 8.11 & $<.005$ & $<.01$ & 3 & 10 \\
\hline & & $8 / 17 / 1993$ & .059 & .227 & 24.2 & 5.9 & $<.005$ & .02 & $<2$ & 8 \\
\hline $3.60648 \mathrm{E}+14$ & SNSC & $8 / 23 / 1993$ & .173 & .232 & 63.9 & 8.01 & $<.005$ & $<.01$ & NS & NS \\
\hline $3.60401 \mathrm{E}+14$ & USGSOS & $8 / 16 / 1993$ & .052 & $<.002$ & 18.5 & 2.61 & $<.005$ & $<.01$ & NS & NS \\
\hline \multirow[t]{2}{*}{$3.60921 \mathrm{E}+14$} & WALLI15 & 4/13/1993 & .141 & .002 & 47.9 & 9.81 & $<.005$ & $<.01$ & NS & NS \\
\hline & & $8 / 21 / 1993$ & .133 & .012 & 42.2 & 8.67 & $<.005$ & $<.01$ & NS & NS \\
\hline
\end{tabular}

Download Comma Separated Values (table5e.csv 3k) 